\title{
Different definitions of the chemical potential in the action with identical partition function in QCD on a lattice
}

\author{
Fabrizio Palumbo 6 \\ INFN - Laboratori Nazionali di Frascati - P. O. Box 13, I-00044 Frascati, ITALIA
}

(February 5, 2020)

\begin{abstract}
It is shown that starting from one and the same transfer matrix formulation of QCD on a lattice, it is possible to obtain both the action of Hasenfratz and Karsch as well as an action where the chemical potential is not coupled to the temporal links.
\end{abstract}

Numerical simulations of QCD at finite baryon density have met with long standing difficultiest. It is then natural to inquire whether the situation cannot be improved by modifying the action used in the path integral. Special attention deserves the coupling of the chemical potential, whose form is due to Hasenfratz and Karsch 2 . They noticed that if in the euclidean path integral of relativistic gauge theories regularized on a lattice the chemical potential is coupled to the fermi fields as in classical systems, specific counterterms are necessary which would be extremely unconvenient in numerical simulations. To avoid this shortcoming, guided by the analogy between chemical potential and temporal gauge field, they coupled the chemical potential in the same way as the gauge fields in the Wilson regularization. They showed that this definition does not require any counterterms, at least in the free theory. After some further analysis 1 , this definition has been accepted as essentially unique for practical purposest.

The feature of the Hasenfratz-Karsh prescription which attracts our attention is the point splitting which implies that the chemical potential must be coupled also to the temporal gauge links. It has a topological consequence, in the sense that only the temporal Polyakov loops give contributions to the path integral which depend on the chemical potential. This looks like a mere lattice artifact, because it does not have any known counterpart in the continuum. But if the interplay between chemical potential and Polyakov loop is an artifact it could be eliminated. This appears an interesting possibility from the point of view of numerical simulations because a term of toplogical nature is expected to affect them significantly.

Therefore we started a systematic investigation of QCD at finite baryon density. We first avoided the use of the chemical potential by considering QCD in a given baryon sectors. The resulting effective action differs by the unconstrained action by terms with a quite different structure with respect to the coupling of the chemical potential in the Hasenfratz-Karsch action. Encouraged by this result we reconsidered the definition of the chemical potential starting from the transfer matrix formalism. The passage to the path integral, as far as the fermion fields are concerned, is based on the insertion of the identity expressed in terms of coherent states in the trace of the transfer matrix. But this can be done in different ways. A possibility which leads exactly to the Hasenfratz-Karsch prescription has been fully investigated 6 . The point splitting and the exponentiation of the chemical potential are in this case a consequence of the praperties of Grassmannian kernels of fermionic operators. But among other possibilities we show here that there is one where there is no point splitting and the chemical potential is not coupled to the temporal links. For Kogut-Susskind fermions in the flavour basis it is not coupled to the gauge fields at all.

We start from the definiton of the grand canonical partition function at finite baryon density according to the ordered product

$$
Z=\operatorname{Tr}\left\{\exp \left(\frac{\mu a_{0}}{T} \hat{Q}_{B}\right) \prod_{n_{0}} \hat{\mathcal{T}}_{q}\left(n_{0}\right)\right\} .
$$

$a_{0}$ is the lattice spacing in the temporal direction, $n_{0}$ the temporal component of the site position vector $n, N_{0}$ is the number of links in the temporal direction, $T$ the temperature

$$
T=\frac{1}{a_{0} N_{0}},
$$

$\hat{Q}_{B}$ is the baryon charge operator and $\hat{\mathcal{T}}_{q}$ is the quark transfer matrix. The pure gauge part of the transfer matrix has been omitted because it does not play any role in the problem. The chemical potential $\mu$ is determined by the condition that the expectation value of the baryon number be

$$
q_{B}=Z^{-1} \operatorname{Tr}\left\{\hat{Q}_{B} \exp \left(\mu N_{0} \hat{Q}_{B}\right) \prod_{n_{0}} \hat{\mathcal{T}}_{q}\left(n_{0}\right)\right\} .
$$

$\hat{\mathcal{T}}_{q}$ can be written $\mathrm{B}$. $\mathrm{E}$ in terms of an auxiliary operator $\hat{T}_{q}\left(n_{0}\right)$

${ }^{*}$ This work has been partially supported by EEC under TMR contract ERB FMRX-CT96-0045 


$$
\hat{\mathcal{T}}_{q}\left(n_{0}\right)=\mathcal{J}^{-1} \hat{T}_{q}^{\dagger}\left(n_{0}\right) \hat{T}_{q}\left(n_{0}+s\right)
$$

where $\mathcal{J}$ is a function of the gauge fields which will be defined later and $s= \pm 1$ for Wilson/Kogut-Susskind fermions respectively. This difference in sign does not reflect any intrinsic difference, but is only due to the different conventions adopted by Lüscher 8 and MontvayMünster 1 , which we maintain for easy reference. The case of Kogut-Susskind fermions will be treated in the flavour basis, because we do not know a suitable formulation of the transfer matrix in the spin diagonal basis. Obviously it is very simple to reexpress the result into this basis, but this introduces awkward nonminimal gauge couplings of the type discussed in $\mathbf{E}$.

$\hat{T}_{q}$ is defined in terms of quark-antiquark creationannihilation operators $\hat{x}^{\dagger}, \hat{y}^{\dagger}, \hat{x}, \hat{y}$ acting in a Fock space. It depends on the time coordinate $n_{0}$ only through the dependence on it of the gauge fields. In fact the creation and annihilation operators do not depend on $n_{0}$. They depend on the spatial coordinates $\mathbf{n}$ of the sites or, in the case of Kogut-Susskind in the flavour basis, of the blocks, and on Dirac, flavour and color indices, $\alpha, f, c$ sometimes comprehensively represented by $I$.

In the transfer matrix formalism often one has to do with quantities at a given (euclidean) time $n_{0}$. For this reason we adopt a summation convention over spatial coordinates and intrinsic indices at fixed time. So for instance we will write

$$
\hat{x}^{\dagger} M\left(n_{0}\right) \hat{x}=\sum_{\mathbf{m}, \mathbf{n}, I, J} \hat{x}_{\mathbf{m}, I}^{\dagger} M_{\mathbf{m}, I ; \mathbf{n}, J}\left(n_{0}\right) \hat{x}_{\mathbf{n}, J},
$$

where $M$ is an arbitrary matrix. In this notation the baryonic charge $\hat{Q}_{B}$ and the auxiliary operator $\hat{T}_{q}\left(n_{0}\right)$ can be written

$$
\hat{Q}_{B}=\hat{x}^{\dagger} \hat{x}-\hat{y}^{\dagger} \hat{y}
$$

$\hat{T}_{q}\left(n_{0}\right)=\exp \left[-\hat{x}^{\dagger} M\left(n_{0}\right) \hat{x}-\hat{y}^{\dagger} M\left(n_{0}\right) \hat{y}\right] \exp \left[\hat{y} N\left(n_{0}\right) \hat{x}\right]$

Their form is the same for Wilson and Kogut-Susskind fermions but the matrices $M$ and $N$ are different in the two cases and will be specified later. The expression of $\hat{T}_{q}\left(n_{0}\right)$ is valid in the gauge $U_{0}=\pi$ which is not admissible. But Menotti and Pelissettd 9 extended the proof of reflection positivity to the gauge $U_{0} \sim \mathbb{1}$ where $U_{0}=\mathbb{1}$ with the exception of a single time slice. In the construction of the path integral formulation of QCD at finite baryon density we do not need to fix the gauge, but to lighten the formalism we will nevertheless put $U_{0}=\mathbb{1}$ and we will reinstate $U_{0}$ in the final result. The reader can check that keeping $U_{0}$ in the intermediate steps one arrives at the same result, provided some care is exercised: for instance when $U_{0} \neq \mathbb{1}$ the expression $\hat{x}^{\dagger} M\left(n_{0}\right) \hat{x}+\hat{y}^{\dagger} M\left(n_{0}\right) \hat{y}$ appearing in Eq. 7 changes and does not commute with $\hat{Q}_{B}$ any longer. We anticipate that $N$ is hermitean and also $M$ is hermitean in the gauge $U_{0}=\mathbb{1}$.
Before transforming the trace into a Berezin integral it is convenient to "distribute" the exponential of the charge operator among the factors in the trace

$$
Z=\operatorname{Tr}\left\{\prod_{n_{0}}\left[\hat{\mathcal{T}}_{q}\left(n_{0}\right) \exp \left(\mu \hat{Q}_{B}\right)\right]\right\} .
$$

Now we introduce between the factors in the above equation the identity

$$
\mathbb{1}=\int\left[d x^{+} d x d y^{+} d y\right] \exp \left(-x^{+} x-y^{+} y\right)|x y><x y|,
$$

where the basis vectors

$$
|x y>=| \exp \left(-x \hat{x}^{\dagger}-y \hat{y}^{\dagger}\right)>
$$

are coherent states and the $x^{+}, x, y^{+}, y$ are Grassmann variables. They will be labeled by the time slice where the unit operator is introduced. For the other indices they are subject to the same convention as the creation and annihilation operators.

Different realizations of the path integral arise depending on the way this insertion is performed. The one leading to the Haseffratz-Karsch prescription has been discussed in detail 5 . Here we consider another possibility based on the following way of writing the exponential of the charge operator

$$
\begin{aligned}
\exp \left(\mu a_{0} \hat{Q}\right) & =\int\left[d x^{+} d x d y^{+} d y\right] \exp (\delta S) \\
& \cdot \exp \left(-x^{+} x-y^{+} y\right)|x y><x y|
\end{aligned}
$$

where

$$
\begin{aligned}
\delta S & =\left(1-\cosh \left(\mu a_{0}\right)\right)\left(x^{+} x+y^{+} y\right) \\
& +\sinh \left(\mu a_{0}\right)\left(x^{+} x-y^{+} y\right) .
\end{aligned}
$$

The above expression is obtained by expanding the exponential of the charge operator, ordering all the annihilation operators to the left of the creation ones and inserting in each monomial the unity between the set of annihilation and the set of creation operators. For the rightmost exponential of the baryon charge one has to move the creation operators to the leftmost position before taking the trace. Replacing the operators by their Grassmannian eigenvalues one gets the above result.

Now the construction of the path integral proceeds in the standard way 8 , yielding the action for zero chemical potential plus the term generated by $\delta S$. To write down this term we must distinguish the case of Wilson fermions from that of Kogut-Susskind.

For Wilson fermions we set $s=1$ in Eq. 1 and assume

$$
\begin{aligned}
& M_{W}\left(n_{0}\right)=-\ln \left((2 K)^{\frac{1}{2}} B^{-\frac{1}{2}}\left(n_{0}\right)\right), \\
& N_{W}\left(n_{0}\right)=2 K B\left(n_{0}\right)^{-\frac{1}{2}} c\left(n_{0}\right) B\left(n_{0}\right)^{-\frac{1}{2}},
\end{aligned}
$$


where $\mathrm{K}$ is the hopping parameter and

$$
\begin{aligned}
B\left(n_{0}\right) & =\mathbb{1}-K \sum_{j=1}^{3}\left(\hat{U}_{j}\left(n_{0}\right) T_{j}^{(+)}+T_{j}^{(-)} \hat{U}_{j}^{+}\left(n_{0}\right)\right) \\
c\left(n_{0}\right) & =\frac{1}{2} \sum_{j=1}^{3} i \sigma_{j}\left(\hat{U}_{j}\left(n_{0}\right) T_{j}^{(+)}-T_{j}^{(-)} \hat{U}_{j}^{+}\left(n_{0}\right)\right) .
\end{aligned}
$$

The link operators $\hat{U}_{\mu}\left(n_{0}\right)$ have the standard Wilson variables $U_{\mu}(n)$ as spatial matrix elements

$$
\left(\hat{U}_{\mu}\left(m_{0}\right)\right)_{\mathbf{m}, \mathbf{n}}=\delta_{\mathbf{m}, \mathbf{n}} U_{\mu}(m) .
$$

We have introduced the translation operators $T_{j}^{( \pm)}$with matrix elements

$$
\left(T_{j}^{( \pm)}\right)_{\mathbf{n}_{1}, \mathbf{n}_{2}}=\delta_{\mathbf{n}_{2}, \mathbf{n}_{1} \pm e_{j}}
$$

$e_{\mu}$ being the unit vectors

$$
\left(e_{\mu}\right)_{\nu}=\delta_{\mu, \nu} .
$$

Next we define the quark field $q$ by the transformation

$$
\begin{aligned}
& x=B^{\frac{1}{2}} P_{0}^{(+)} q, \quad y=B^{\frac{1}{2}} P_{0}^{(-)} q \\
& \bar{q}=q^{\dagger} \gamma_{0}
\end{aligned}
$$

where

$$
P_{0}^{( \pm)}=\frac{1}{2}\left(1 \pm \gamma_{0}\right) .
$$

The jacobian of this transformation is the function $\mathcal{J}$ introduced in Eq. 4 . The partition function takes the form

$$
Z_{W}=\int[d \bar{q} d q] \exp \left(S_{W}+\delta S_{W}\right),
$$

where

$$
\begin{aligned}
S_{W}= & \sum_{n}\left\{K \sum _ { \mu } \left[\bar{q}(n)\left(1+\gamma_{\mu}\right) U_{\mu}(n) q\left(n+e_{\mu}\right)\right.\right. \\
& \left.\left.+\bar{q}\left(n+e_{\mu}\right)\left(1-\gamma_{\mu}\right) U_{\mu}^{\dagger}(n) q(n)\right]-\bar{q}(n) q(n)\right\}
\end{aligned}
$$

is the Wilson action with Wilson parameter $r=1$ and zero chemical potential and the contribution of the chemical potential is obtained from Eq. 12

$$
\begin{aligned}
\delta S_{W} & =\sum_{n_{0}}\left\{\overline { q } ( n _ { 0 } ) \left[\left(1-\cosh \left(\mu a_{0}\right)\right)\right.\right. \\
& \left.\left.+\sinh \left(\mu a_{0}\right) \gamma_{0}\right] B\left(n_{0}\right) q\left(n_{0}\right)\right\} .
\end{aligned}
$$

The first term breaks the chiral symmetry, but this is only a consequence of the breaking by the Wilson term. Indeed we will see that the corresponding term for KogutSusskind fermions respects the chiral invariance. Notice the "plus" sign in the exponential of the action, to comply with Lüscher's convention.

The case of Kogut-Susskind fermions will studied only in the flavour basis. The $n_{\mu}$ are now the block coordinates and the gauge fields are defined on the block links. We set $s=-1$ in Eq. 因 and assumel

$$
\begin{aligned}
& M_{K S}\left(n_{0}\right)=0 \\
& N_{K S}\left(n_{0}\right)=\left\{\sum _ { j = 1 } ^ { 3 } \left[\gamma_{5} \otimes t_{5} t_{j}+\gamma_{j}\left(P_{j}^{(-)} \hat{U}_{j}\left(n_{0}\right) T_{j}^{(+)}\right.\right.\right. \\
& \left.\left.\left.-P_{j}^{(+)} T_{j}^{(-)} \hat{U}_{j}^{+}\left(n_{0}\right)\right)\right]+\frac{m}{K} \mathbb{1} \otimes \mathbb{1}+\gamma_{5} \otimes t_{5} t_{0}\right\},
\end{aligned}
$$

where $m$ is the quark mass parameter and $\mathrm{K}$ the hopping parameter. In the tensor product the $\gamma$-matrices act on Dirac indices, while the $t$-matrices

$$
t_{\mu}=\gamma_{\mu}^{T}
$$

act on flavor indices. The projection operators $P_{\mu}^{( \pm)}$are given by

$$
P_{\mu}^{( \pm)}=\frac{1}{2}\left[\mathbb{1} \otimes \mathbb{1} \pm \gamma_{\mu} \gamma_{5} \otimes t_{5} t_{\mu}\right] .
$$

The quark field $q$ is obtained by the transformation

$$
\begin{aligned}
& x=4 \sqrt{K} P_{0}^{(+)} q, y^{+}=4 \sqrt{K} P_{0}^{(-)} q \\
& \bar{q}=q^{\dagger} \gamma_{0}
\end{aligned}
$$

whose jacobian is the function $\mathcal{J}$ introduced in Eq. A. The partition function takes the form

$$
Z_{K S}=\int[d \bar{q} d q] \exp \left[-16\left(S_{K S}+\delta S_{K S}\right)\right]
$$

where

$$
\begin{aligned}
S_{K S} & =\frac{1}{2} K\left\{\sum_{n} \bar{q}(n)\left(\gamma_{\mu} \otimes \mathbb{1}-\gamma_{5} \otimes t_{5} t_{\mu}\right) U_{\mu}(n) q\left(n+e_{\mu}\right)\right. \\
& -\bar{q}(n)\left(\gamma_{\mu} \otimes \mathbb{1}+\gamma_{5} \otimes t_{5} t_{\mu}\right) U_{\mu}^{\dagger}\left(n-e_{\mu}\right) q\left(n-e_{\mu}\right) \\
& \left.+2 \bar{q}(n) \gamma_{5} \otimes t_{5} t_{\mu} q(n)+a m \bar{q}(n) \mathbb{1} \otimes \mathbb{1} q(n)\right\}
\end{aligned}
$$

is the Kogut-Susskind action with zero chemical potential and the chemical potential contribution is obtained from Eq. 12

$$
\begin{aligned}
\delta S_{K S}= & -K\left\{\sum _ { n } \overline { q } ( n ) \left[\left(1-\cosh \left(\mu a_{0}\right)\right) \gamma_{5} \otimes t_{5} t_{0}\right.\right. \\
& \left.\left.+\sinh \left(\mu a_{0}\right) \gamma_{0} \otimes \mathbb{1}\right] q(n)\right\} .
\end{aligned}
$$

The factor 16 in front of the action accounts for the fact that the volume element with Kogut-Susskind fermions is 16 times larger than in the Wilson case.

Let us emphasize that the partition functions with the present action and with that of Hasenfratz and Karsch are identical with one another. It is worth while checking this identity in the free case and comparing to the "naive" 
definition. For simplicity we will consider only the Wilson case neglecting the mass and the spatial Wilson term. But notice that we cannot omit also the temporal one, because otherwise we cannot construct the transfer matrix. We then consider the quark matrix

$$
Q=\gamma_{0}\left(a_{0} \nabla_{0}+\sigma\right)+a_{0} \gamma \cdot \nabla+\frac{1}{2} a_{0}^{2} \square_{0}+1-R
$$

where

$$
\begin{aligned}
& \left(\nabla_{\mu} f\right)(n)=\frac{1}{2 a_{\mu}}\left[f\left(n+e_{\mu}\right)-f\left(n-e_{\mu}\right)\right] \\
& \left(\square_{\mu} f\right)(n)=\frac{1}{a_{\mu}}\left[f\left(n+e_{\mu}\right)+f\left(n-e_{\mu}\right)-2\right]
\end{aligned}
$$

and

$$
\begin{aligned}
& \sigma=\sinh \left(\mu a_{0}\right), \quad R=\cosh \left(\mu a_{0}\right), \text { new definition } \\
& \sigma=\mu a_{0}, \quad R=1, \quad \text { "naive" definition. }
\end{aligned}
$$

We now evaluate the energy density $\mathcal{E}$ at zero temperature, setting $a_{\mu}=a$. Normalizing at zero baryon density

$$
\mathcal{E}=-\frac{1}{2 \pi^{3} a^{4}} \int_{-\pi}^{\pi} d^{3} q s^{2}[\mathcal{I}(\mu)-\mathcal{I}(0)],
$$

where

$$
s^{2}=\sum_{j}\left(1-\cos q_{j}\right)
$$

$$
\begin{aligned}
\mathcal{I}(\mu) & =\frac{1}{2 \pi} \int_{-\pi}^{\pi} d q_{0}\left[\left(\sin q_{0}-i \sigma\right)^{2}+s^{2}+\left(\cos q_{0}-R\right)^{2}\right]^{-1} \\
& =\frac{1}{2 \sqrt{A^{2}-B^{2}}}[1-\theta(R-A)] .
\end{aligned}
$$

$\theta$ is the step function. With the new definition

$$
\begin{aligned}
R-A & =\frac{1}{2}\left[s^{2}+2(1-\cosh (\mu a)] \sim \frac{1}{2}\left[s^{2}-(\mu a)^{2}\right]\right. \\
A^{2}-B^{2} & =s^{2}\left[1+\frac{1}{4} s^{2}\right]
\end{aligned}
$$

while with the "naive" definition

$$
\begin{aligned}
R-A & =\frac{1}{2}\left[s^{2}-(\mu a)^{2}\right] \\
A^{2}-B^{2} & =s^{2}\left[1+\frac{1}{4} s^{2}-\frac{1}{2}(\mu a)^{2}+\frac{(\mu a)^{4}}{4 s^{2}}\right] .
\end{aligned}
$$

Therefore in the "naive" case the energy density has a quadratic divergence, while with the new definition

$$
\mathcal{E}=\frac{2}{\pi^{2}} \mu^{4}
$$

is equal to the value obtained by the Hasenfratz-Karsch prescription in the presence of the temporal Wilson term (without the spatial one).

In conclusion we have shown how starting from one and the same transfer matrix we can realize identical path integrals with different actions for QCD at finite baryon density. In the action constructed in the present work the chemical potential is not coupled to the temporal links, in contrast to the Hasenfratz-Karsh prescription. It might therefore have quite different properties in numerical simulations which it could be interesting to investigate.
${ }^{1}$ J.B.Kogut, hep-lat/0208077; R.Aloisio, V.Azcoiti, G.DiCarlo, A.Galante and A.F.Grillo, Nucl. Phys. B564 (2000) 489 X.Q.Luo, E.Gregory, S.Guo and H.Kroger, hepph/0011120; Z.Fodor and S.D.Katz, hep-lat/0106002; Ph. de Forcrand and O.Philipsen, hep-lat/0205016; M.D'Elia and M.-P.Lombardo, hep-lat/0209146

${ }^{2}$ P.Hasenfratz and F.Karsh, Phys. Lett.125B (1983) 308; J.Kogut, M.Matsuoka, M.Stone,H.W.Wyld, J.H.Shenker, J.Shighemitsu and D.K.Sinclair, Nucl. Phys. B225[FS9] (1983) 93

3 N.Bilic and R.V.Gavai, Z. Phys. 23C (1984) 77; R.V.Gavai, Phys. Rev. D32 (1985) 519

${ }^{4}$ I.Montvay and G.Münster, Quantum fields on a lattice, Cambridge University Press, 1994

${ }^{5}$ F.Palumbo, hep-lat/0202021, Nucl. Phys. 643B (2002) 391; hep-lat/0208002, Nucl. Phys. B, to be published

${ }^{6}$ V.K.Mitryushkin, hep-lat/0206024

${ }^{7}$ M.Creutz, Foundations of Phys.30 (2000) 487

${ }^{8}$ M.Lüscher, Commun.math.Phys. 54 (1977) 283

${ }^{9}$ P.Menotti and A.Pelissetto, Comm. Math. Phys. 113 (1987) 369 